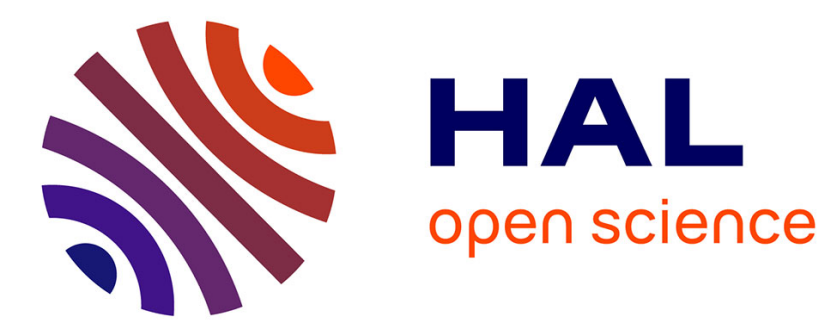

\title{
Three-dimensional stability of a horizontally sheared flow in a stably stratified fluid
}

\author{
Axel Deloncle, Jean-Marc Chomaz, Paul Billant
}

\section{To cite this version:}

Axel Deloncle, Jean-Marc Chomaz, Paul Billant. Three-dimensional stability of a horizontally sheared flow in a stably stratified fluid. Journal of Fluid Mechanics, 2007, 570 (January), pp.297-305. 10.1017/s0022112006003454 . hal-01023344

HAL Id: hal-01023344

https://hal-polytechnique.archives-ouvertes.fr/hal-01023344

Submitted on $20 \mathrm{Jul} 2014$

HAL is a multi-disciplinary open access archive for the deposit and dissemination of scientific research documents, whether they are published or not. The documents may come from teaching and research institutions in France or abroad, or from public or private research centers.
L'archive ouverte pluridisciplinaire HAL, est destinée au dépôt et à la diffusion de documents scientifiques de niveau recherche, publiés ou non, émanant des établissements d'enseignement et de recherche français ou étrangers, des laboratoires publics ou privés. 


\title{
Three-dimensional stability of a horizontally sheared flow in a stably stratified fluid
}

\author{
By AXEL DELONCLE, JEAN-MARC CHOMAZ \\ AND PAUL BILLANT \\ LadHyX, CNRS, Ecole Polytechnique, 91128 Palaiseau Cedex, France \\ axel.deloncle@ladhyx.polytechnique.fr
}

(Received 7 July 2006 and in revised form 9 October 2006)

This paper investigates the three-dimensional stability of a horizontal flow sheared horizontally, the hyperbolic tangent velocity profile, in a stably stratified fluid. In an homogeneous fluid, the Squire theorem states that the most unstable perturbation is two-dimensional. When the flow is stably stratified, this theorem does not apply and we have performed a numerical study to investigate the three-dimensional stability characteristics of the flow. When the Froude number, $F_{h}$, is varied from $\infty$ to 0.05 , the most unstable mode remains two-dimensional. However, the range of unstable vertical wavenumbers widens proportionally to the inverse of the Froude number for $F_{h} \ll 1$. This means that the stronger the stratification, the smaller the vertical scales that can be destabilized. This loss of selectivity of the two-dimensional mode in horizontal shear flows stratified vertically may explain the layering observed numerically and experimentally.

\section{Introduction}

Because of its importance in industrial and geophysical applications, the evolution of shear flows has been much studied (Ho \& Huerre 1984). The basic case of a flow in a homogeneous fluid is well known. Squire's theorem (Squire 1933) states that any threedimensional unstable mode is less unstable than two-dimensional modes, implying that shear flows are dominated by two-dimensional instabilities. Necessary conditions for their existence are given by Rayleigh's inflection point criterion (Rayleigh 1887) and Fjørtoft's criterion (Fjørtoft 1950) on the basic velocity profile in the inviscid case. Howard (1961) also showed that the temporal growth rate of two-dimensional perturbations are confined inside a semi-circle in the complex plane.

Much attention has also been devoted to the situation of a vertical shear in a stably stratified fluid. Miles (1961) and Howard (1961) established a sufficient condition for stability as $R i>1 / 4$ with $R i=N^{2} / S^{2}$, the Richardson number, where $N$ is the Brunt-Väisälä frequency and $S$ the shear rate.

In contrast, the case of a non-vertical shear in a vertically stratified fluid has been little addressed. Blumen (1970) extended the application of Howard's semicircle theorem for hydrostatic perturbations in a stratified fluid for an arbitrary orientation of the shear.

However, the Squire theorem does not hold for such flows, meaning that it is not at present known whether two-dimensional perturbations dominate the dynamics of a stratified sheared flow. In order to address this question, we investigate the 

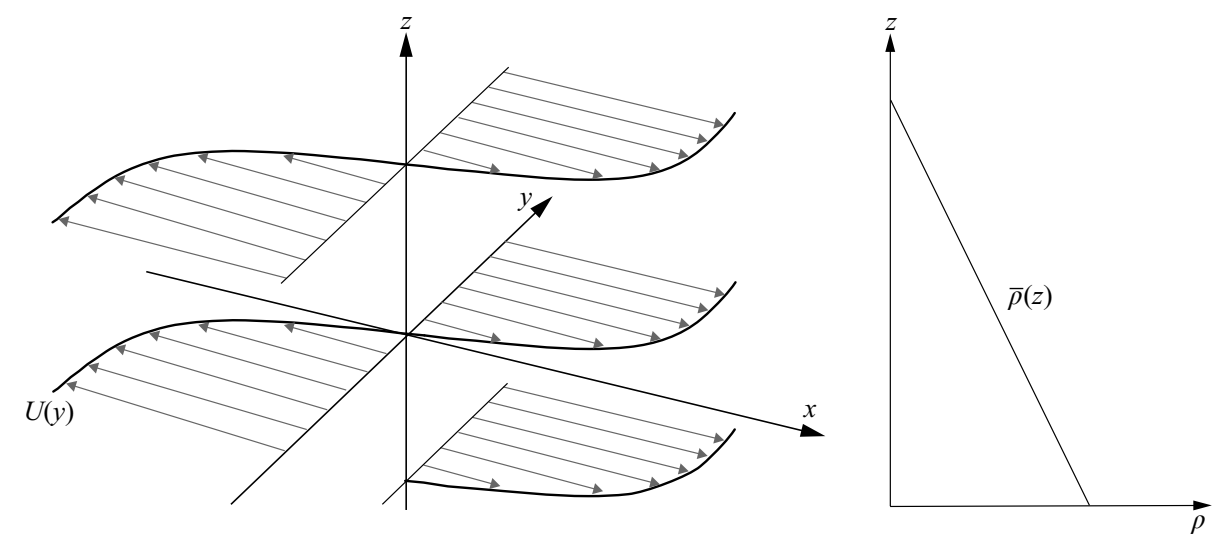

FIGURE 1. Sketch of the base flow. The hyperbolic-tangent velocity profile is horizontal with a velocity in the $x$-direction sheared in the $y$-direction and uniform in the $z$-direction. The fluid is stably stratified in the vertical $z$-direction.

three-dimensional linear stability of a purely horizontal mixing layer, the hyperbolictangent velocity profile, in a vertically stratified fluid.

The paper is organized as follows. In $\S 2.1$, the governing equations as well as the basic state are presented. In $\S \S 2.2$ and 2.3, we give the linearized equations and outline the numerical method. Section 3 is devoted to the results for different stratifications. Concluding remarks follow in $\S 4$.

\section{Problem formulation}

\subsection{The governing equations and the basic state}

The flow is assumed to be inviscid non-diffusive stably stratified and to satisfy the Boussinesq approximation for the Euler equations:

$$
\begin{gathered}
\frac{\mathrm{D} \boldsymbol{u}}{\mathrm{D} t}=-\frac{1}{\rho_{0}} \nabla p+b \boldsymbol{e}_{z}, \\
\nabla \cdot \boldsymbol{u}=0, \\
\frac{\mathrm{D} b}{\mathrm{D} t}+N^{2} w=0,
\end{gathered}
$$

where $\boldsymbol{u}=(u, v, w)$ is the velocity vector in Cartesian coordinates, $\rho_{0}$ a constant reference density, $p$ the pressure, $b=-g \rho^{\prime} / \rho_{0}$ the buoyancy with $\rho^{\prime}$ the density perturbation with respect to the mean density $\bar{\rho}, g$ the gravity and $\boldsymbol{e}_{z}$ the unit vector in the upward $z$-direction. $N=\sqrt{-g / \rho_{0} \mathrm{~d} \bar{\rho} / \mathrm{d} z}$ is the Brunt-Väisälä frequency assumed here to be constant.

We investigate the stability of a purely horizontal flow, the hyperbolic-tangent velocity profile whose shear is in the $y$-direction (figure 1):

$$
\boldsymbol{U}(y)=U(y) \boldsymbol{e}_{x}=U_{0} \tanh \left(\frac{y}{L}\right) \boldsymbol{e}_{x},
$$

where $U_{0}$ and $L$ are, respectively, the velocity and length scales of the base flow and $\boldsymbol{e}_{x}$ is the unit vector in the $x$-direction.

In the following, length and time scales are non-dimensionalized such that $L=1$ and $U_{0}=1$. The basic state becomes $U(y)=\tanh y$. For simplicity, the same notation is kept for the dimensionless variables. 


\subsection{Linearized equations}

Since the basic state is uniform along the $x$ - and $z$-axis, we consider infinitesimal perturbations of the form

$$
[\tilde{\boldsymbol{u}} ; \tilde{p} ; \tilde{b}](x, y, z, t)=[\boldsymbol{u} ; p ; b](y) \exp \left(\mathrm{i} k_{x} x+\mathrm{i} k_{z} z+\sigma t\right)+\text { c.c. },
$$

where $\sigma$ is the growth rate, $k_{x}$ and $k_{z}$ the wavenumbers along directions $x$ and $z$, and c.c. denotes the complex conjugate.

For unbounded velocity profiles, the boundary conditions correspond to vanishing perturbations at infinity:

$$
\lim _{y \rightarrow \pm \infty}[\boldsymbol{u} ; p ; b](y)=[0 ; 0 ; 0] .
$$

The linearized equations for the perturbation quantities can be written in terms of $u$, $v$ and $b$ by eliminating the vertical velocity $w$ and the pressure $p$ :

$$
\boldsymbol{A}\left(\begin{array}{l}
u \\
v \\
b
\end{array}\right)=\sigma \boldsymbol{B}\left(\begin{array}{l}
u \\
v \\
b
\end{array}\right)
$$

where

$$
\begin{aligned}
\boldsymbol{A} & =\left(\begin{array}{ccc}
0 & \mathrm{i} k_{x}\left\{U\left(\mathrm{D}^{2}-k^{2}\right)-\mathrm{D}^{2} U\right\} & \mathrm{i} k_{z} \mathrm{D} \\
\mathrm{i} k_{x}(\mathrm{D} U+U \mathrm{D}) & \mathrm{D}^{2} U+k_{x}^{2} U+\mathrm{D} U \mathrm{D} & 0 \\
\mathrm{i} k_{x} / F_{h}^{2} & \mathrm{D} / F_{h}^{2} & k_{x} k_{z} U
\end{array}\right), \\
\boldsymbol{B} & =\left(\begin{array}{ccc}
0 & k^{2}-\mathrm{D}^{2} & 0 \\
-\mathrm{D} & \mathrm{i} k_{x} & 0 \\
0 & 0 & \mathrm{i} k_{z}
\end{array}\right),
\end{aligned}
$$

and where $k^{2}=k_{x}^{2}+k_{z}^{2}, \mathrm{D}=\mathrm{d} / \mathrm{d} y$ and $F_{h}=U_{0} / L N$ is the horizontal Froude number. This matrix form is convenient for solving the problem numerically.

\subsection{Numerical procedure}

The three-dimensional stability of the hyperbolic-tangent profile is determined by computing all the eigenvalues of the matrix operator (2.7).

Because the domain of study $y \in]-\infty, \infty[$ is unbounded, an algebraic transformation $\tilde{y}=y / \sqrt{1+y^{2}}$ is first used to map the infinite interval of $y$ to a finite one $\tilde{y} \in]-1,1[$. The two operators $\boldsymbol{A}$ and $\boldsymbol{B}$ are then discretized using a set of $Q$ Chebyshev polynomials interpolated on Gauss-Lobatto collocation points. The algebraic mapping in conjunction with the Chebyshev polynomials leads to the so-called rational Chebyshev functions (Grosch \& Orszag 1977; Boyd 2001).

The discretized version of the eigenvalue problem (2.7) is finally solved by calling the eigensolver eig of Matlab. To eliminate the spurious modes which are not physically relevant, two discretizations with different numbers $Q_{1}=297$ and $Q_{2}=369$ of Chebyshev polynomials are used. We keep only the eigenvalues whose relative variation between the two discretizations is less than 0.01 .

\section{Results}

To describe the general properties of the stability problem, it is more convenient to obtain a single equation for $v$ from the linearized equations:

$$
\mathrm{D}\left(\frac{\mathrm{D} v}{\tilde{k}^{2}}\right)-\left[\frac{\mathrm{i} k_{x}}{s} \mathrm{D}\left(\frac{\mathrm{D} U}{\tilde{k}^{2}}\right)+1\right] v=0,
$$

where $s=\sigma+\mathrm{i} k_{x} U$ and $\tilde{k}^{2}=k_{x}^{2}+F_{h}^{2} s^{2} k_{z}^{2} /\left(F_{h}^{2} s^{2}+1\right)$. 

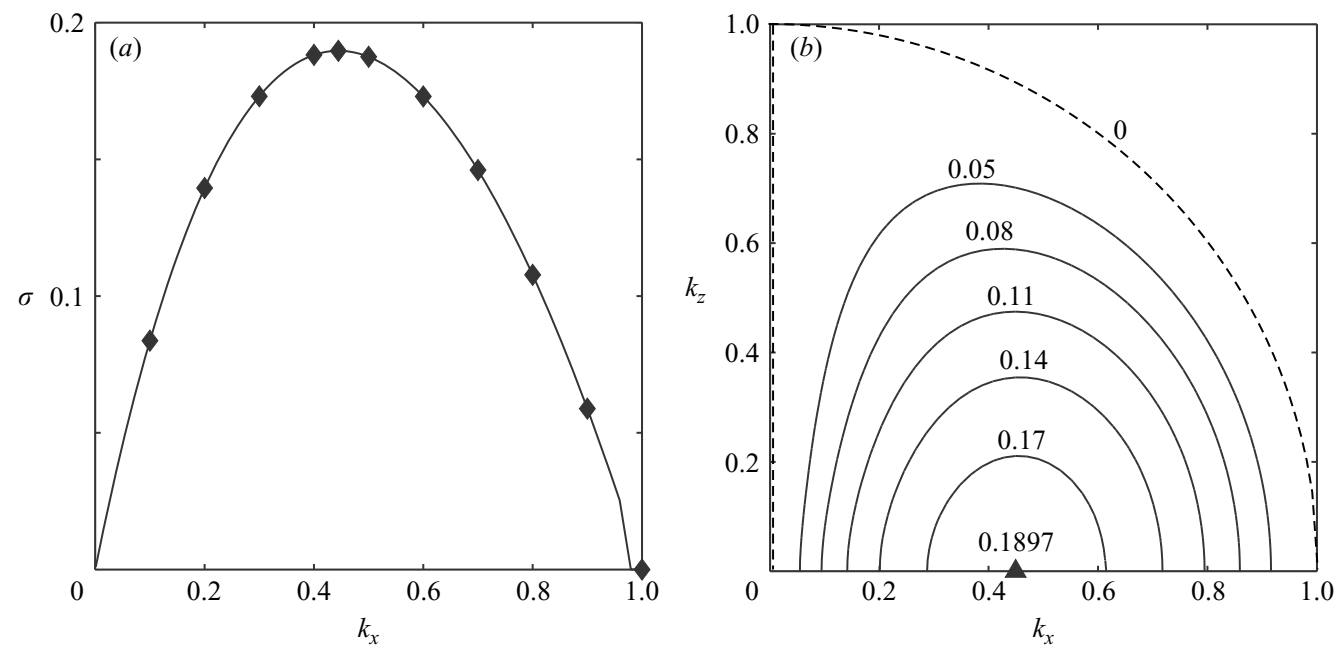

FIGURE 2. Homogeneous fluid. (a) Growth rate $\sigma$ of a two-dimensional disturbance as a function of its wavenumber $k_{x}$; the results of Michalke (1964) are also plotted with $\bullet$. $(b)$ Contours of growth rate $\sigma$ of a three-dimensional disturbance as a function of its horizontal $k_{x}$ and vertical $k_{z}$ wavenumbers; $\boldsymbol{\Delta}$ indicates the most unstable mode. The unstable domain is bounded by neutral modes deduced from the Squire's transformation and plotted as a dashed line.

First, we can see that if $\sigma$ is an eigenvalue of (3.1) for given wavenumbers $\left(k_{x}, k_{z}\right)$, then it is also an eigenvalue for $\left(k_{x},-k_{z}\right)$ while $-\sigma$ is an eigenvalue for $\left(-k_{x}, k_{z}\right)$. Therefore, positive $k_{x}$ and $k_{z}$ only can be studied without loss of generality.

When the fluid is homogeneous $\left(F_{h}=\infty\right)$, we have $\tilde{k}^{2}=k^{2}$ so that equation (3.1) reduces to the Rayleigh equation

$$
\mathrm{D}^{2} v-\left[\frac{\mathrm{i} k_{x}}{s} \mathrm{D}^{2} U+k^{2}\right] v=0 .
$$

We see from equation (3.2) that the growth rate can be sought in the form $\sigma=k_{x} f(k)$, where $f(k)$ is a function of $k=\sqrt{k_{x}^{2}+k_{z}^{2}}$ only. This implies the wellknown Squire transformation (Schmid \& Henningson 2001), i.e. the growth rate and the eigenmode of a three-dimensional perturbation $k_{z} \neq 0$ can be deduced from that of a two-dimensional perturbation $k_{z}=0$. It implies also that any threedimensional disturbance with wavenumbers $\left(k_{x}, k_{z}\right)$ having a growth rate $\sigma^{3 D}$ has a corresponding two-dimensional disturbance $\left(\sqrt{k_{x}^{2}+k_{z}^{2}}, 0\right)$ with a larger growth rate $\sigma^{2 D}=\sigma^{3 D} \sqrt{k_{x}^{2}+k_{z}^{2}} / k_{x}$. Since the hyperbolic tangent profile is neutral for wavenumbers $\left(k_{x}=1, k_{z}=0\right)$ the Squire theorem also implies that all perturbations with $k_{x}^{2}+k_{z}^{2}=1$ are neutral. In the homogeneous case, it is also possible to prove the Rayleigh criterion from (3.2); a necessary condition of instability is that the velocity profile $U(y)$ has an inflection point, i.e. $\mathrm{D}^{2} U$ changes sign.

Figure $2(a)$ shows the growth rate of a two-dimensional disturbance as a function of its horizontal wavenumber $k_{x}$ in the case of a homogeneous fluid. These results are in agreement with those of Michalke (1964) (diamonds in figure 2a). In particular, the growth rate is purely real with a maximum amplification, $\sigma_{\max }=0.1897$, occurring at $k_{x \max }=0.4449$. The relative error does not exceed $1 \%$ except for the neutral mode around $k_{x}=1$. This slight difference is due to the presence of a regular singularity at $y=0$ in the neutral eigenmodes at $k_{x}=1$. As our primary interest is the effect of the 
stratification on unstable modes, we have made no provision for keeping track of these singularities. The general case of a three-dimensional disturbance in a homogeneous fluid has been computed directly and is presented in figure $2(b)$. The most unstable mode is two-dimensional, in agreement with the Squire's transformation. The unstable domain in the $\left(k_{x}, k_{z}\right)$-plane is inside the neutral curve $k_{x}^{2}+k_{z}^{2}=1$ (dashed line) in agreement with the Squire transformation.

In the stratified case, the classical theorems are not valid. The Squire transformation no longer applies for equation (3.1) in the case $F_{h} \neq \infty$ so that three-dimensional modes may possibly be more unstable than two-dimensional modes. In the stratified case, equation (3.1) shows that the Rayleigh inflection point criterion is only a necessary condition for two-dimensional perturbation, but not for three-dimensional perturbations. This leaves open the possibility for a horizontally sheared flow without an inflection point to be unstable to three-dimensional modes, but such a counter example to the Rayleigh criterion has not yet been found. In any case, the three-dimensional stability properties cannot be inferred from the two-dimensional results when the flow is stratified; they have to be computed numerically.

The task is slightly more involved than in the homogeneous case when $F_{h} \neq \infty$ because other regular singularities are present when $s= \pm \mathrm{i} / F_{h}$ and $s= \pm \mathrm{i} k_{x} /\left(F_{h} k\right)$ in addition to the singularity at $s=0$. In non-dimensional form, these extra singularities correspond to a Doppler shifted frequency $\mathrm{i} \sigma-k_{x} U$ equal to the Brunt-Väisälä frequency and to the frequency of internal gravity waves, respectively. Moreover, it can be shown that the two solutions of equation (3.1) are analytic at the critical point $s= \pm \mathrm{i} k_{x} /\left(F_{h} k\right)$, but only one solution is analytic at $s= \pm \mathrm{i} / F_{h}$. These additional singularities might explain why the computations become difficult and require higher spatial resolution for small growth rates when the fluid is stratified.

Figure 3 is similar to figure $2(b)$, but in stratified cases. The figure shows the growth rate contours in the $\left(k_{x}, k_{z}\right)$-plane for Froude numbers from $F_{h}=10$ down to $F_{h}=0.05$. In all four cases, the most amplified mode remains two-dimensional as in the homogeneous case. However, by paying attention to the vertical wavenumber range, we see that the unstable domain expands towards extremely large $k_{z}$ as $F_{h}$ decreases making the unstable domain strongly anisotropic when $F_{h}$ is small. Moreover, we see that the contours in figures $3(b)-3(d)$ have the same shape and differ only by their vertical wavenumber range, suggesting a self-similarity law.

The growth rate for the most unstable streamwise wavenumber $k_{x \max }=0.4449$ are plotted as a function of $k_{z}$ in figure $4(a)$ for different stratifications $F_{h}$. The curve of $\sigma$ as a function of $k_{z}$ widens dramatically for small $F_{h}$. This implies that the selectivity of the two-dimensional modes strongly decreases with $F_{h}$, meaning that a broad range of vertical scales can develop in such a flow. For example, the growth rate for $F_{h}=0.05$ at $k_{z}=10$ is only $20 \%$ smaller than that for the two-dimensional mode.

Figure $4(b)$ presents the same growth rate curve as figure 4(a), but plotted versus $F_{h} k_{z}$. As already anticipated from figure 3, the growth rate contours collapse when represented as a function of $F_{h} k_{z}$. The growth rates for $F_{h}=0.1$ and $F_{h}=0.05$ are indiscernible. This self-similarity implies that the growth rate is a function of $F_{h} k_{z}$ and not $F_{h}$ and $k_{z}$ separately, i.e. $\sigma\left(k_{x}, k_{z}, F_{h}\right)=\tilde{\sigma}\left(k_{x}, F_{h} k_{z}\right)$ for $F_{h} \leqslant 1$. This result is a generic feature valid for any strongly stratified flow as shown by Billant \& Chomaz (2001).

For strong stratifications, this self-similarity may be proved for the neutral curve. First, we can see in equation (3.1) that the modes along the $k_{x}=0$ axis remain neutral. Moreover, for strong stratifications $\left(F_{h} \ll 1\right)$, we have at leading order $\tilde{k}^{2} \simeq k_{x}^{2}+F_{h}^{2} s^{2} k_{z}^{2}$, 

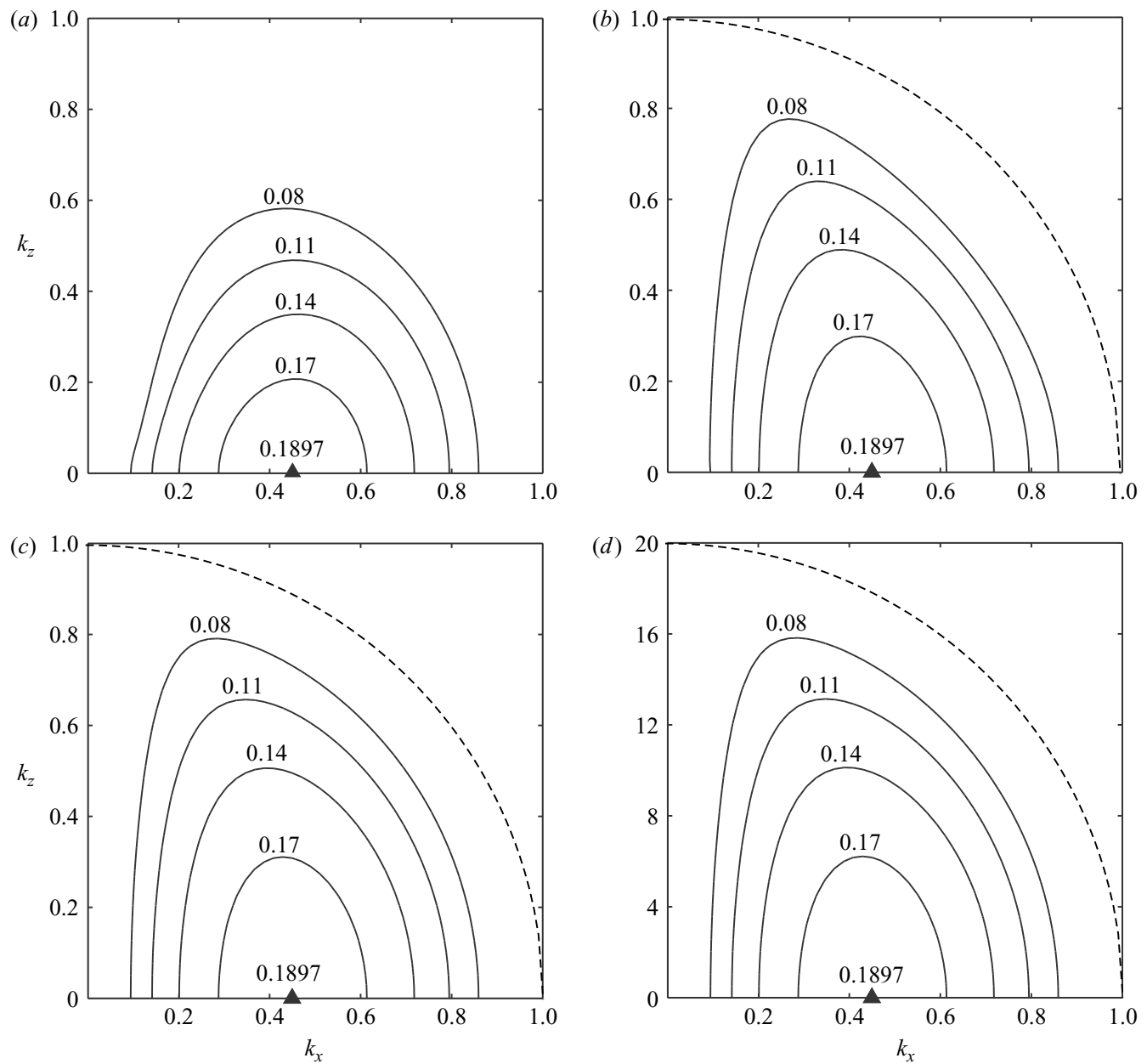

FIGURE 3. As figure $2(b)$, but in a stratified fluid. Increasing stratifications are presented: (a) $F_{h}=10,(b) F_{h}=1,(c) F_{h}=0.1$ and $(d) F_{h}=0.05$. The boundaries of the unstable domain valid for strong stratifications $\left(F_{h} \ll 1\right)$ are also plotted as a dashed line for $(b)-(d)$.

which is equivalent to the hydrostatic approximation. Following Blumen (1970), the neutral modes $(\sigma=0)$ of equation (3.1) are then:

$$
v=k_{x} \operatorname{sech}^{k_{x}^{2}} y,
$$

where $k_{x}$ verifies the condition:

$$
k_{x}^{2}+\left(F_{h} k_{z}\right)^{2}=1 .
$$

The latter condition is similar to the one valid in a homogeneous fluid $k_{x}^{2}+k_{z}^{2}=1$, except that the vertical wavenumber has been rescaled by $F_{h}$. The boundaries of the unstable domain are plotted with dashed lines in figures $3(b)-3(d)$.

\section{Conclusion}

We have investigated the three-dimensional instability of the hyperbolic tangent velocity profile. In homogeneous fluid, the Squire theorem tells us that 

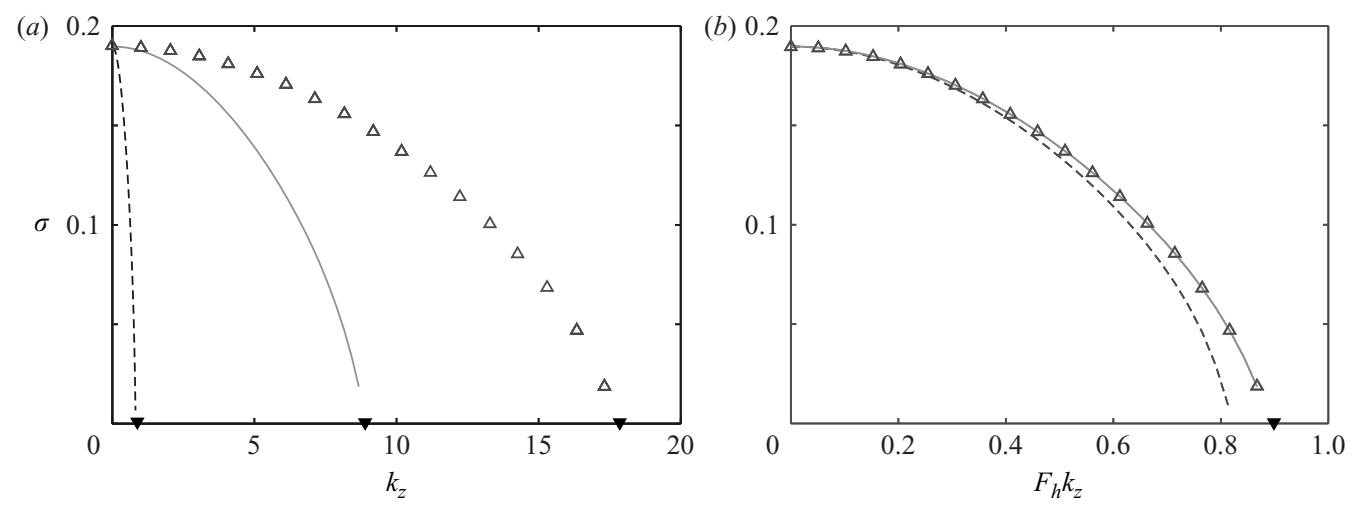

FIGURE 4. Growth rate as a function of $(a)$ the vertical wavenumber $k_{z}$ and $(b)$ the rescaled vertical wavenumber $F_{h} k_{z}$ for the most unstable two-dimensional wavenumber $k_{x \max }=0.4449$. Three stratifications are plotted; ---, $F_{h}=1 ;-, F_{h}=0.1 ; \triangle, F_{h}=0.05$. The neutral modes given by equation (3.4) for strong stratifications are also plotted with $\boldsymbol{\nabla}$.

three-dimensional perturbations are always less unstable than two-dimensional ones. When the shear is horizontal and the fluid is vertically stratified, the theorem does not apply and we have determined numerically the three-dimensional stability of this particular shear flow.

Five different Froude numbers $F_{h}$ have been studied, from a homogeneous fluid to a strongly stratified fluid. In all cases, the most unstable mode remains twodimensional, but the stratification has been found to destabilize a broad range of three-dimensional modes whose extent increases in inverse proportion to $F_{h}$. Both the numerical results and an asymptotic analysis show that the unstable domain in the $\left(k_{x}, k_{z}\right)$-plane becomes strongly anisotropic with a neutral boundary that is no longer a circle $k_{x}^{2}+k_{z}^{2}=1$ as in the homogeneous case, but an elongated ellipse $k_{x}^{2}+\left(F_{h} k_{z}\right)^{2}=1$. When $F_{h} \leqslant 1$, numerical results show that the growth rate converges to a universal curve $\sigma\left(k_{x}, k_{z}, F_{h}\right)=\tilde{\sigma}\left(k_{x}, F_{h} k_{z}\right)$ function of the rescaled vertical wavenumber $F_{h} k_{z}$ rather than $F_{h}$ and $k_{z}$ separately. This decrease of the characteristic vertical length as $F_{h}$ is in agreement with the self-similarity found by Billant \& Chomaz (2001). In practice, our analysis shows that all the three-dimensional modes with $k_{z}$ smaller than $1 / F_{h}$ have a growth rate similar to the two-dimensional modes.

The selectivity of the two-dimensional instability is therefore dramatically decreased when the stratification is increased. This property might explain the results of the numerical simulations of Basak \& Sarkar (2006) where a horizontal mixing layer is initially perturbed by a large-amplitude noise. The flow evolves into pancake vortices with a vertical correlation length scale that decreases with stratification. This vertical scale selection is reminiscent of the increase of the width of the unstable vertical wavenumber band predicted by the present stability analysis when $F_{h}$ decreases. Indeed three-dimensional perturbations with a wavenumber in the $x$-direction equal to the most unstable two-dimensional wavenumber, but with a vertical wavenumber $k_{z}$ scaling as $1 / F_{h}$, will grow as fast as the two-dimensional perturbation. Such a perturbation will induce a vertical deformation of the shear layer out of phase every half wavelength $\pi / k_{z}$ on the vertical and therefore leads to a roll-up resulting in a pattern of vortices staggered on the vertical very similar to those observed in Basak \& Sarkar (2006). 


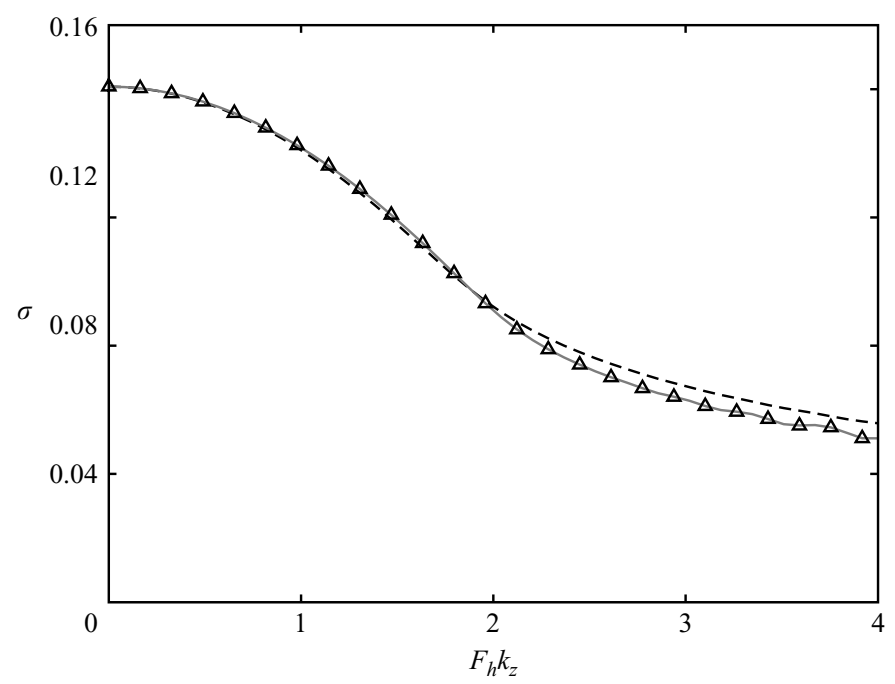

FIGURE 5. Similar to figure $4(b)$ for the Bickley jet $U(y)=\operatorname{sech}^{2} y$. Growth rate as a function of the rescaled vertical wavenumber $F_{h} k_{z}$ for the most unstable two-dimensional wavenumber $k_{x \max }=0.9021$. Key as for figure 4 . Only the leading mode, at present even in $y$, is considered. The odd mode is not presented, but follows a similar trend.

The layering observed by Praud, Fincham \& Sommeria (2005) in the early evolution of the flow produced by towing a rake of vertical flat plates might result from a similar destabilization of large vertical wavenumber for the primary instability of the sheared flow and not from a secondary instability of the primary vortices.

Oceanic currents or atmospheric jets are configurations where horizontal shear is observed (Pedlosky 1982) and the present analysis questions the frequent assumption that such horizontal shear will be unstable to a two-dimensional (barotropic) mode. Instead, we may imagine that the weak selectivity for the two-dimensional mode demonstrated here for stratified flows will favour the formation of vertically decorrelated layers of thickness proportional to $F_{h}$. This conjecture is confirmed by the stability analysis of the Bickley jet. Figure 5 presents the instability growth rate of this flow versus the rescaled vertical wavenumber at the horizontal wavenumber corresponding to the most unstable two-dimensional mode (similar to figure $4 b$ ). The instability growth rate converges toward a unique curve as a function of the rescaled vertical wavenumber $F_{h} k_{z}$ in agreement with the proposed self-similarity.

It is interesting to contrast this case of a horizontal shear with the well-known situation of a vertical shear in a stably stratified fluid. As recalled in $\S 1$, strong stratification stabilizes vertical shear flow since the associated Richardson number is then larger than $1 / 4$, whereas it destabilizes horizontal shear flow versus perturbations with short vertical wavelength. This suggests that in most realistic situations, a shear flow will be sensitive mainly to the horizontal component of the shear rather than to the vertical one because of this opposing effect of stratification.

\section{REFERENCES}

BASAK, S. \& SARkar, S. 2006 Dynamics of a stratified shear layer with horizontal shear. J. Fluid Mech. 568, 19-54.

Billant, P. \& Chomaz, J. M. 2001 Self-similarity of strongly stratified inviscid flows. Phys. Fluids 13, 1645-1651. 
Blumen, W. 1970 Hydrostatic neutral waves in a parallel shear flow of a stratified fluid. J. Atmos. Sci. 28, 340-344.

Boyd, J. P. 2001 Chebyshev and Fourier Spectral Methods, 2nd edn. Dover.

FJøRTOFT, R. 1950 Application of integral theorems in deriving criteria for instability for laminar flows and for the baroclinic circular vortex. Geofys. Publ. Oslo 17 (6), 1-52.

Grosch, C. E. \& OrszaG, S. A. 1977 Numerical solution of problems in unbounded regions: coordinates transforms. J. Comput. Phys. 25, 273-296.

Ho, C. M. \& Huerre, P. 1984 Perturbed shear layers. Annu. Rev. Fluid. Mech. 16, 365-424.

Howard, L. N. 1961 Note on a paper of John W. Miles. J. Fluid Mech. 10, 509-512.

Michalke, A. 1964 On the inviscid instability of the hyperbolic tangent velocity profile. J. Fluid Mech. 19, 543-556.

Miles, J. W. 1961 On the stability of heterogeneous shear flows. J. Fluid Mech. 10, 496-508.

Pedlosky, J. 1982 Geophysical Fluid Dynamics. Springer.

Praud, O., Fincham A. M. \& Sommeria, J. 2005 Decaying grid turbulence in a strongly stratified fluid. J. Fluid Mech. 522, 1-33.

RaYleigh, Lord 1887 On the stability of certain fluid motions. Proc. Math. Soc. Lond. 11, 57-70.

Schmid, P. J. \& Henningson, D. S. 2001 Stability and Transition in Shear Flows. Springer.

SQuire, H. B. 1933 On the stability for three-dimensional disturbances of viscous fluid flow between parallel walls. Proc. Math. Soc. Lond. A 142, 621-628. 